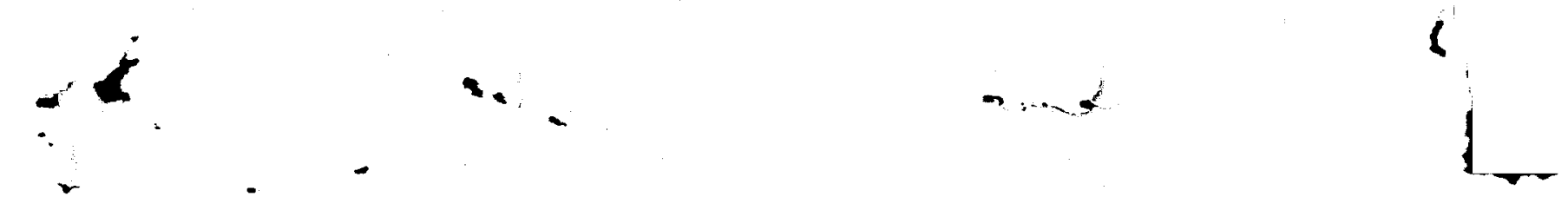

\title{
BAPSDLISHED PRELIMINARY DATI
}

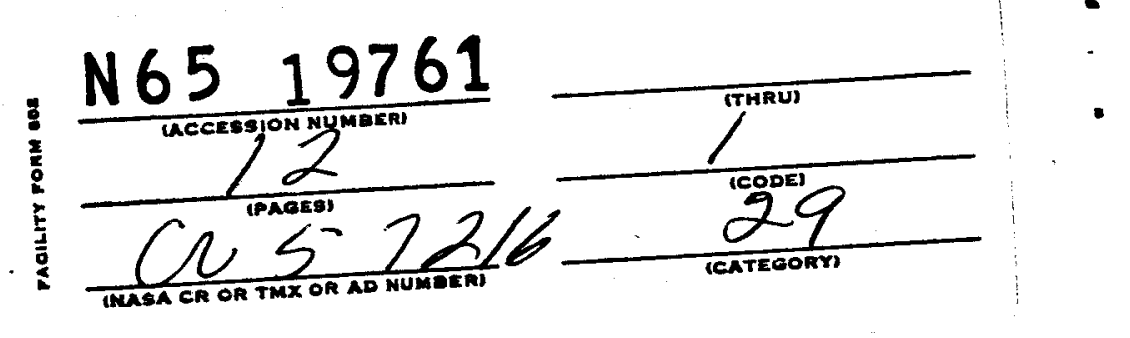

The Fermi Acceleration of Charged Particles*

in the Transition Region

Beyond the Magnetosphere

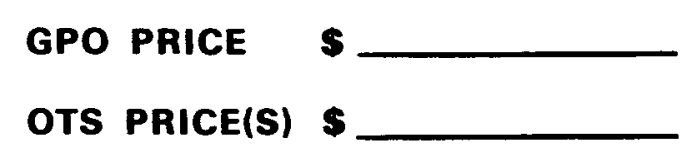

December 24, 1964

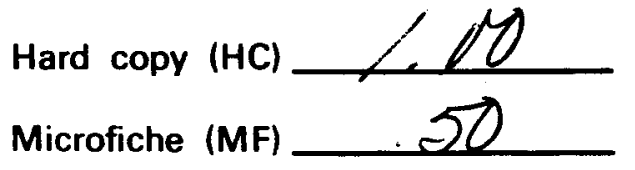

\section{S. Shen}

Physics Department, Purdue University

Lafayette, Indiana

and

Department of Space Science and Applied Physics

The Catholic University of America

Washington, D. C.

and

C. C. Chang

Department of Space Science and Applied Physics

The Catholic University of America

Washington, D. C.

*This work is done under partial support of National Aeronautics and Space Administration. 
Eividence of the existence of high energy electrons with energy $E \geqslant 30 \mathrm{Kev}$ in the transition region beyond the magnetosphere was reported recently by Fan, Gloeckler and Simpson 2 and also by Anderson, Harris and Paoli . As there can be no possibility of prolonged geomagnetic trapping in this region, the origin of these high energy electrons is a rather interesting problem. We suggest that, because of the characteristic geometry and distorted structure of the interplanetary magnetic field near the magnetopause in the transition region, these electrons are locally accelerated by means of a Parker-Wentzel version of the Germi mechanism. $3,4,5$ The general picture is 6 illustrated in Figure I-a, a modification of Ness, et al(Fig. 40). In the transition region II which starts at the shock front, the solar wind and magnetic field can be considered as frozenin and both are influenced by the downstream magnetosphere. The solar wind compresses interplanetary magnetic field against the magnetosphere. The field strength increases with distance. 6

Also, according to Ness it was observed to be turbulent and unsteady with finite amplitude. The 7 unsteadiness can be considered as magnetohydrodynamic waves propagating toward the downstream magnetopause. Because of the low density, the waves suffer no dissipation until they meet each other and build up a large amplitude pulse. The total field strength along the field lines inside the transition region may be expressed as

$$
B(z, t)=B_{0}(z, t)+\sum_{i} H_{i}(z) \delta\left(z-z_{i}-l_{i} t\right)
$$

Where $Z$ is the distance measured downstream from shock front along the line of force, $B_{0}(Z, t)$ is the background magnetic field, whose strength in general increases as $Z$ increases and depends weakly on the time t. That is, the interplanetary magnetic field is compressed against the magnetopause (CF.Fig. I-a and Fig. I-b). Also $Z_{i}$ is the position of the ith magnetic pulse with strength $H_{i}(Z)$ at $t=0$. And $U_{i}$ is the parellel component of Alfven wave velocity of the traveling magnetic pulse 
along the field line based approximately on $B_{0}$. In such a collisionless region, the thickness of these large amplitude hydromagnetic waves is expected to be of the order of the Larmor radius of a thermal ion, 7 which is much larger than the electron's Larmor radius. Thus the local electrons with pitch angles larger than $\tan ^{-1}\left[\frac{B_{0}(Z)}{H_{i}(Z)}\right]^{\frac{1}{2}}$ will be trapped by the advancing pulse and be pushed toward the stronger magnetic field. The equation of motion for a charged particle in an adiabatically varying magnetic field is

$$
\frac{d}{d t}\left(m v_{11}\right)=-\mu \frac{\partial B(z, t)}{\partial z}
$$

Here, $V_{I I}$ is the parallel (to the field line) component velocity of the particle and $\mu=\frac{W_{\perp}}{B}$, is an adiabatic invariant where $W_{\perp}$ is the transverse component of the particles kinetic energy.

Equation (2) indicates, as is well known, that the longitudinal motion of a particle in an adiabatically varying field is mathematically equivalent to that of the motion of a particle under the force of a one dimensional potential $\mu B(z, t)$.$] In our case, \mu$ being an invariant, the potential is related to two parts of the magnetic field. One part is a "hill", i.e., the background magnetic field $B_{0}$, and the other part is a series of barriers, i.e., the magnetic pulses $H_{i}(Z)$, which move up the hill with velocity $U_{i}$. Note that the transverse kinetic energy of the particle is identical with the potential $\mu \mathrm{B}$. Therefore the particle's kinetic energy in this one dimensional model is the actual longitudinal kinetic energy $W_{\| 1}$ in the three dimensional space, while its potential energy is the actual transverse kinetic energy. $W_{\mathcal{L}}$. 
$-3-$

The collision of the particle with the advancing pulse pushes the particle up the hill until all its kinetic energy is converted into the potential energy, then the particle starts to move backward and meets with the pulse again. Each collision increases the particle's energy by an amount of $4 V_{\| l} U+4 U^{2}$. Because the pulse is moving up the hill toward stronger field, a part of this energy is transformed to potential energy.

The collision process repeats itself until the particle's kinetic energy becomes larger than the pulse's or "barrier's" height, at the point $Z_{2}, W_{11}\left(Z_{2}\right) \geq \mu H\left(Z_{2}\right)$ then the particle will roll over the crest of the pulse and go down the hill. Let $Z_{1}$ be the point where the particle was first trapped by the pulse. We have

$$
\begin{aligned}
& W_{1}\left(z_{2}\right)=\mu B_{0}\left(z_{2}\right)=\frac{B_{0}\left(z_{2}, t_{2}\right)}{B_{0}\left(z_{1}, t_{1}\right)} W_{\perp}\left(z_{1}\right) \\
& W_{11}\left(z_{2}\right) \geq \mu H\left(z_{2}\right)=\frac{H\left(z_{2}, t_{2}\right)}{B_{0}\left(z_{1}, t_{1}\right)} W_{\perp}\left(z_{1}\right)
\end{aligned}
$$

On the other hand, the particle can be trapped by the pulse at $Z_{1}$, only if

$$
\frac{w_{1}\left(z_{1}\right)}{w_{11}\left(z_{1}\right)} \geq \frac{B_{0}\left(z_{1}, t_{1}\right)}{H\left(z_{1}, t_{1}\right)}
$$

Combine (3), (4) and (5) gives

$$
W\left(z_{2}\right) \geq \frac{B_{0}\left(z_{2}, t_{2}\right)+H\left(z_{2}, t_{2}\right)}{B_{0}\left(z_{1}, t_{1}\right)+H\left(z_{1}, t_{1}\right)} W\left(z_{1}\right)
$$

Equation (6) gives the energy gain of the particle in a single trip. 
$\cdot-4-$

Consider $Z_{1}$ to be some point in the outer transition region and $Z_{2}$ to be some point along the background magnetic lines where the magnetic field increases in strength near the shoulder of the magnetopause. We may assume some values of $B_{0}$ and $H$ consistent with Explorer 18 measurements; $B_{0}\left(z_{1}\right) \sim 7 \gamma_{1} H\left(z_{1}\right) \sim 6 \gamma_{1} B_{0}\left(z_{2}\right) \sim 30 \gamma$ and $H\left(\mathbf{z}_{2}\right) \sim 7 \gamma$

This gives $\quad W\left(z_{2}\right) \approx 3 W\left(z_{1}\right)$. Z

Clearly it is unlikely that electrons trapped only once can be accelerated to $30 \mathrm{KeV}$ energy.

Now consider the situation after the particle rolls over the crest of the pulse; as it moves down the hill, its transverse kinetic energy is converted into longitudinal kinetic energy. Without some kind of effective redistribution mechanism of the longitudinal energy, the particle just rolls backward over successive pulses and can hardly be trapped again. We propose that superimposed on $B_{0}$ are small scale magnetic turbulence or irregularities due to local current loops. These irregularities scatter electrons and randomize their energy distribution. The transfer of energy by scattering from one component to another is proportional to the degree of anistropicity. Paker ${ }^{8}$ also found out that scattering efficiency diminishes as the scale of irregularity becomes either large or small compared to the Larmor radius of the particle and is maximum when the scale is comparable to the Larmor radius. Thus, whether the particle can be retrapped or not depends on the density of irregularities or the average scale of the magnetic eddies. If the condition $\frac{W_{\perp}}{W_{11}}>\frac{B}{H}$ can be restablished before the particle escapes from the transition region (i.e. across the shock boundary), the particle will again be trapped by the wave train of advancing pulses and be pushed up to the stronger field. Under ideal conditions this 
process of acceleration and redistribution will repeat itself and boost up the electron's kinetic energy until its Larmor radius becomes comparable to the thickness of the magnetic pulse, which is estimated to be of the order of the Larmor radius of a thermal ion ( $W \sim 1 \mathrm{Kev})$. This gives the electron energy an upper limit of $2 \times 10^{3} \mathrm{Kev}$.

The time required to boost an electron's energy to $30 \mathrm{Kev}$ can also be estimated. The electrons are trapped by the magnetic pulse in the outer portion of the transition region and penetrate the pulse near the magnetopause. Thus the distance traveled by the pulse is $10,000 \mathrm{Km}$. Assume the propagation velocity of the pulse is $\sim 100 \mathrm{Km} / \mathrm{sec}$ (the Alfven velocity in the transition region), then it takes 100 sec. to triple the electron's energy. Therefore, for an electron with initial energy $X$ in ev, $10^{2} \log _{3}\left(\frac{3 \cdot 10^{4}}{X}\right)$ seconds are needed to increase its energy to $30 \mathrm{Kev}$. For example, to reach $30 \mathrm{Kev}$ energy a $10 \mathrm{ev}$ electron has to be trapped 7 times and the time required is $\sim 10$ minutes.

In the above calculation we have assumed $\mu$ to be a constant as the particle was trapped ahead of the magnetic pulse and advancing toward stronger field. The scattering of the small scale field with the particle will change the value of $\mu$ and, in general,, increases the energy gain per trip. An elaborate calculation included this effect and the effect of the attenuation of large amplitude waves was carried out and will be pulbished elsewhere. However, the inclusion of these factors do not affect the basic assumptions and the major consequences of the proposed acceleration process. They may be listed as follows:

Basic assumptions:

1. There are large amplitude waves propagating toward the magnetopause causing the trapping of electrons. 


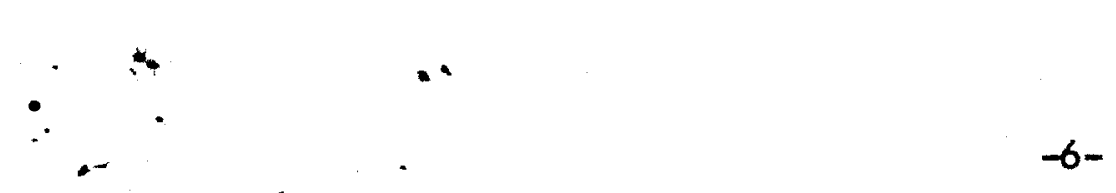

2. There are small scale magnetic irregularities or turbulences causing the redistribution of the electron's energy.

3. The interplanetary magnetic field in the transition region is, in general, stronger near the magnetopause through compression than in the outer portion of the region.

\section{Major Consequences:}

I. Only electrons are accelerated.

2. The accelerated electrons tend to bunch in the magnetic valleys because the trapping regions are ahead of the wave.

3. The velocity distribution of the accelerated electrons is anisotropic.

4. The intensity of the high energy electrons is closely correlated with the degree of distrubance of the magnetic field in the transition region (the evidence of the presence of large amplitude wave). After the transition region is hit by a magnetic storm, there should be a time lag of the order of a few minutes before any increase in the intesity of high energy electrons.

5. The accelerated electrons may leak out of the shock front to interplanetary space immediately after magnetic storm. They may also be pushed over the "hill" (the bottleneck part of the field shown in Fig. (-a) and leak to the dark side of the earth.

Comparison with the existing experimental measurement 1,2 indicates good qualitative agreement for consequences (1), (2), (4) and (5). The angular distribution of the high energy electrons, as far as we known, has yet to be measured. So there is no comparison for consequence (3) now. However, we think this is the most interesting consequence. According to the proposed mechanism, after the particle has been pushed up the "hill" and is about to penetrate the magnetic pulse at $Z^{\prime}$, (Cf. Fig. 2) $W_{11} \approx \frac{H\left(z^{\prime}\right)}{B\left(z^{\prime}\right)} W_{\perp}$ and 


\section{$-7-$}

$\tan ^{2} \quad \theta \approx \frac{B\left(z^{\prime}\right)}{H\left(z^{\prime}\right)}>1$. But after it penetrates the pulse and moves down the hill, most of its energy is transformed to the longitudinal component. Conservation of energy gives $W_{11}\left(Z^{\prime \prime}\right) \approx$

$\frac{H\left(z^{\prime}\right)+B\left(z^{\prime}\right)-B\left(z^{\prime \prime}\right)}{H\left(z^{\prime}\right)+B\left(z^{\prime}\right)} \underset{\text { and } \tan ^{2} \theta \approx \frac{B\left(z^{\prime \prime}\right)}{H\left(z^{\prime}\right)+B\left(z^{\prime}\right)-B\left(z^{\prime \prime}\right)}}{\text { As it moves further away from the magnetopause toward the outer transition region the }}$.

scattering with small scale irregularities gradually smears out the asymetry and $\tan ^{2} \theta$

approaches unit.

A measurement of the pitch angle along the field line would strongly indicate the correctness of our conjecture. 


\section{References}

1. C. Y. Fan, G. Gloeckler, and J. A. Simpson. Phys. Rev. letter 13, 149,(1964).

2. K. A. Anderson, H. K. Harris, and R. J. Paoli, Univ. California, Berkley Report, Vo. 5, Issue 50 .

3. E, Fermi, Phys Rev. 75 1169, (1949).

4. E. N. Parker, Phys Rev. 109 1328, (1958)

5. D. G. Wentzel, Astrophys, J. 137 135, (1963)

6. N. F. Ness, C. S: Scearce, and J. B. Seek, J. Geophys. Res. 69 3531, (1964).

7. E. N. Parker, Astrophys J. $129 \quad 217$ (1959)

8. E. N. Parkẹr, J. Geophys. Res. 69, 1755, (1964) iPhys. Rev. Letters 14, 55(1965)

9. J.R. Jokipii and L. Davis Jr. Phys. Rev. Letters I3 739 (1964) 


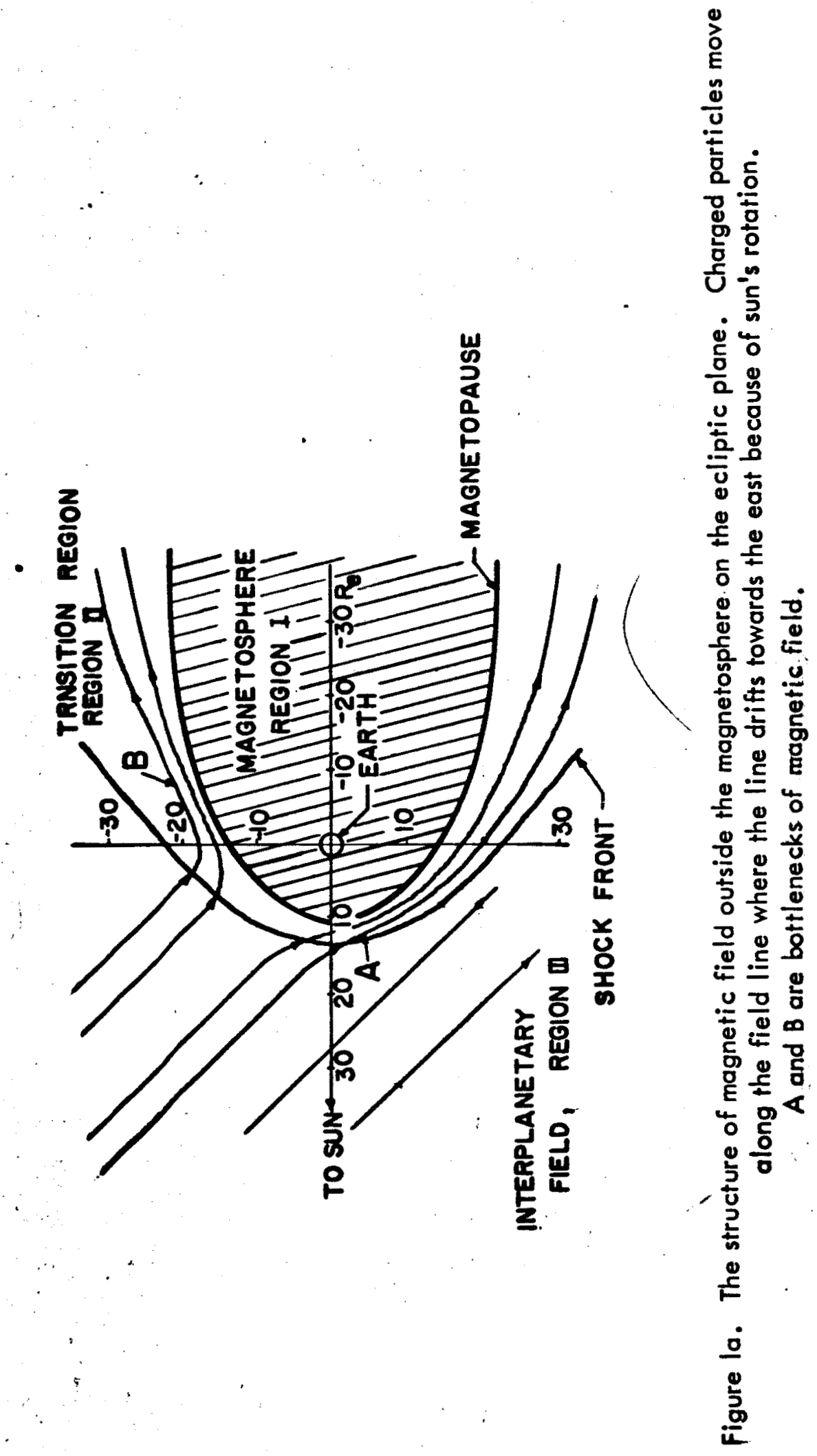




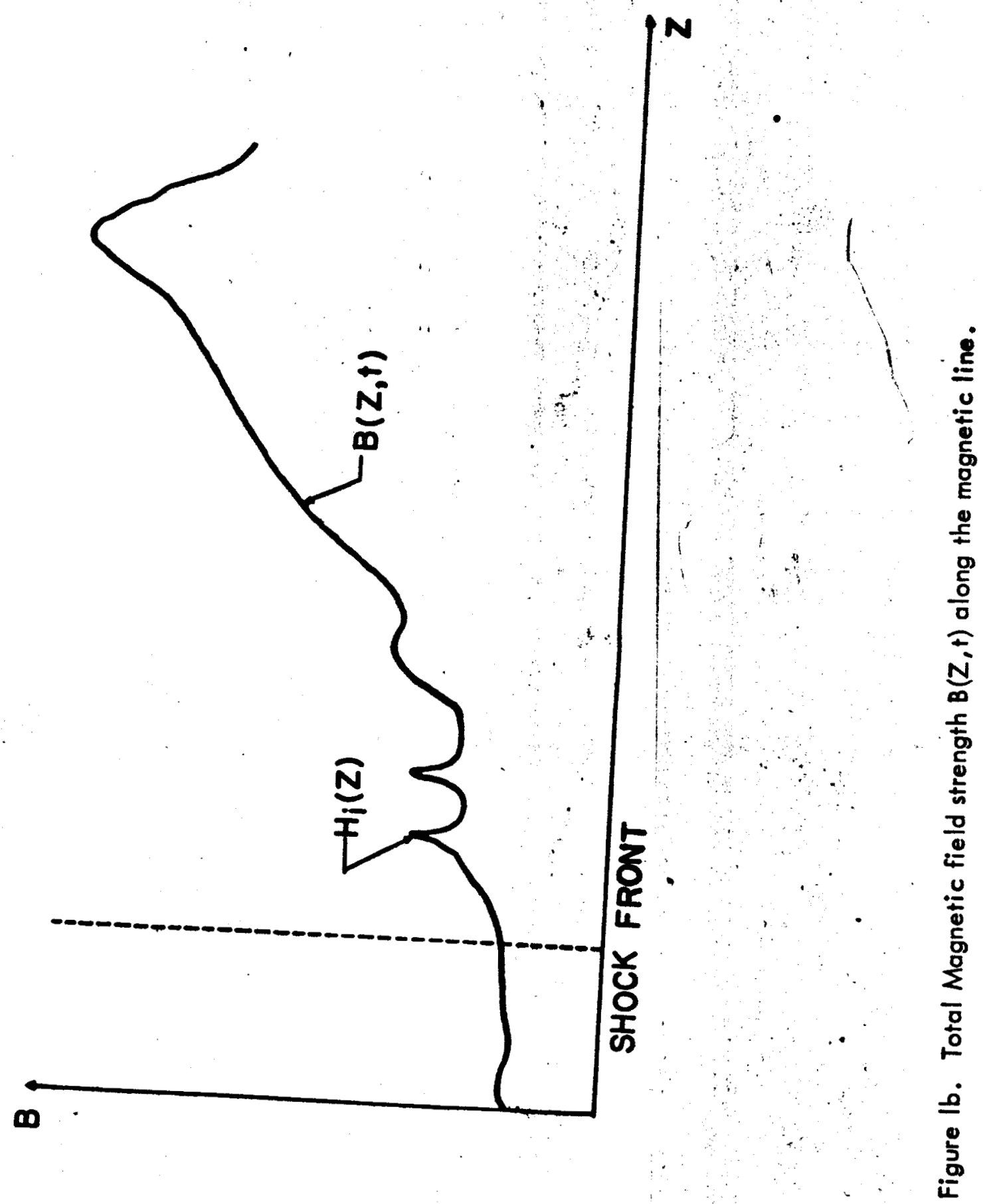


$\therefore \because$

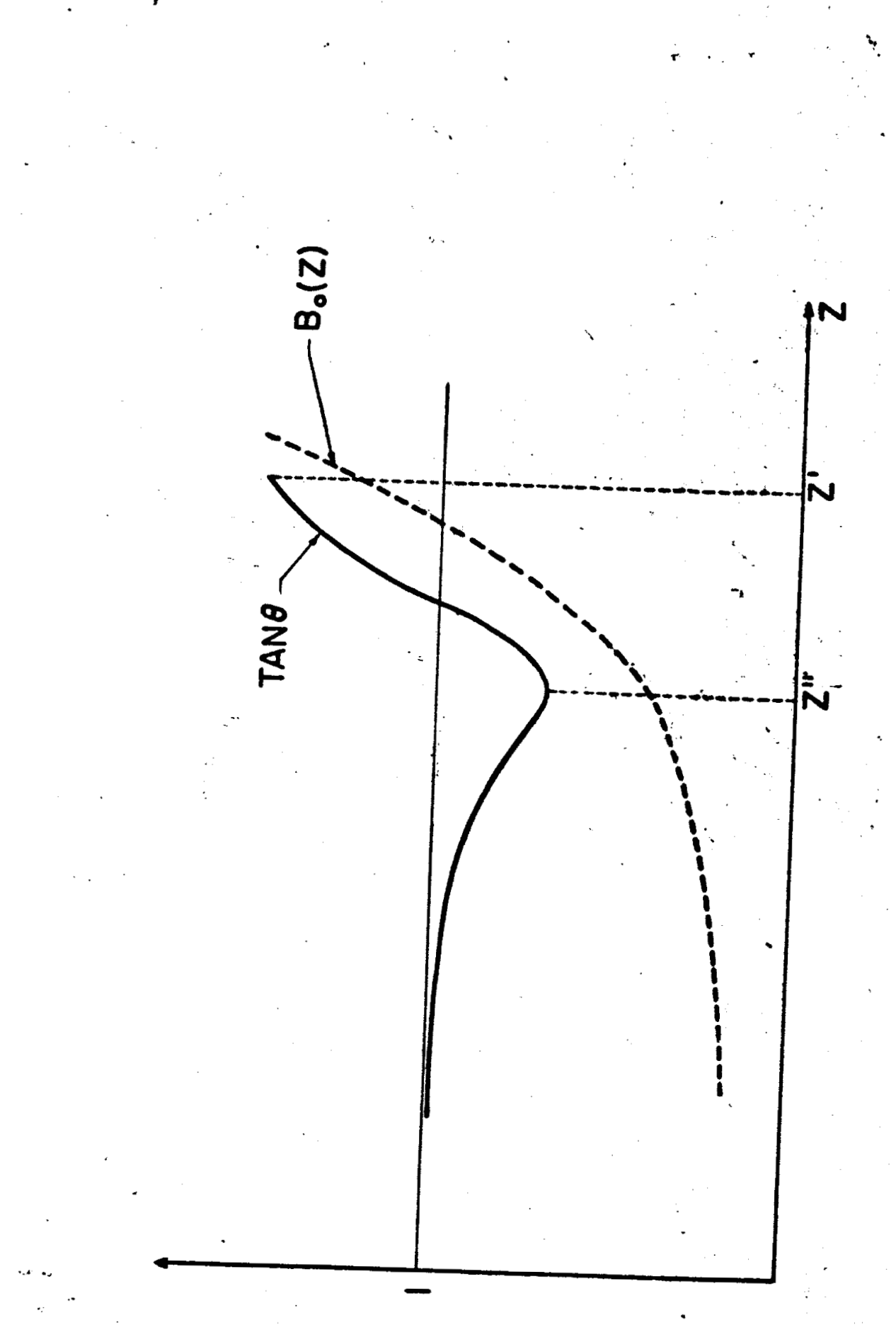

들

$\mp$

음

N

$\infty^{9}$

\begin{tabular}{l}
0 \\
0 \\
0 \\
0 \\
0 \\
0 \\
\hline 0 \\
\hline \\
\hline \\
\hline
\end{tabular}

$\Phi$

$\frac{\mathscr{D}}{\mathbf{0}}$

旁

范

동

용

- 